\title{
Gentamicin in Pregnancy: Seeing past the drug categorisation in pregnancy
}

\section{R. Chean $1,2,3,4$, S.M. Garland $3,4,5,6$, L. Leung 7}

${ }^{1}$ Infectious Diseases physician, Department of Medicine, Latrobe Regional Hospital

2 Department of Microbiology, Dorevitch Pathology

${ }^{3}$ Department of Microbiology and Infectious Diseases, Royal Women's Hospital

${ }^{4}$ Department of Microbiology, Royal Children's Hospital

${ }^{5}$ Murdoch Childrens Research Institute

${ }^{6}$ Department of Obstetrics and Gynaecology, University of Melbourne, Parkville

${ }^{7}$ Department of Pharmacy, Royal Women's Hospital

Gentamicin's well-described role in the treatment of sepsis extends to that for

pregnant women, with the literature articulating its many indications in the

treatment of infections in obstetrics ${ }^{1}$. Despite sepsis causing significant mortality

and morbidity to both the gravid woman and the fetus/ newborn ${ }^{2}$, its use is

likely overcautious with many clinicians reluctant to use this antibiotic in septic

obstetric patients in Australia.

Gentamicin is an aminoglycoside antibiotic used in the treatment of sepsis owing especially to its role in combating Gram-negative infections. It has favourable pharmacological characteristics of concentration-dependent killing, rapid action,

prolonged post-antibiotic effect ${ }^{1}$, and improved clinical outcomes when used in This is the author manuscript accepted for publication and has undergone full peer review but has not been through the copyediting, typesetting, pagination and proofreading process, which may lead to differences between this version and the Version of Record. Please cite this article as doi: 10.1111/imj.13315

This article is protected by copyright. All rights reserved. 
combination therapy in patients with septic shock ${ }^{3}$. The low aminoglycoside resistance amongst Gram-negative bacteria in Australia further underscores its role in sepsis management ${ }^{4}$. In addition, the physiology of pregnancy also likely accounts for the lack of toxicity in gravid women ${ }^{1,2}$. Indeed, gentamicin is intrinsically woven into the protocol management of sepsis in obstetrics patients managed at the Royal Women's Hospital.

We postulate however that the drug categorisation in pregnancy of ' $\mathrm{D}$ ' for gentamicin may lead to hesitancy in prescribing gentamicin in pregnant women, even when clinically indicated. This categorisation states that it belongs to medicines that are suspected to have caused or may be expected to cause human fetal malformations or irreversible damage. This however is overly simplistic, misleading and doesn't effectively communicate the risk, or lack thereof, gentamicin has on pregnancy.

This risk categorisation of gentamicin in pregnancy has been based on case reports of totally irreversible bilateral congenital deafness in children on streptomycin, and small animal studies of nephrotoxicity from gentamicin administration $^{5}$. In fact, cases of gentamicin use during pregnancy leading putatively to fetal ototoxicity have been only rarely described 2,5 , whilst work that involve population studies or randomised trials had shown that gentamicin use during pregnancy had not resulted in any adverse events to gravid patients nor to their fetuses. ${ }^{1,5,6}$ 
The US FDA recently adopted progressive amendments to its regulations governing the content and format of "pregnancy" labelling of human prescription drugs and biological products ${ }^{7}$. It has removed the pregnancy categories labelling of $A, B, C, D$, and $X$ of these products and in its place is the Pregnancy and Lactation Labelling Rule (PLLR) or Final Rule that requires changes to how information is presented in prescription medicine. This change allows clinicians and their patients to make informed decisions based on available detailed information rather than a single oversimplified categorisation of a medicine regarding the risk during pregnancy.

We agree that such an approach might help both clarify the role gentamicin has and demystify its potential safety concerns in antenatal sepsis. Whilst an amendment to the pregnancy drug categorisation may not be imminent in Australia, doctors and health care providers locally need to be cognisant of the critical benefits of gentamicin in severe sepsis including in pregnancy. This benefit can be attained when gentamicin is used appropriately and expediently for severe sepsis in pregnancy with for example a daily dosing of $4-7 \mathrm{mg} / \mathrm{kg}$, and for the shortest period of time till antimicrobial susceptibility of the offending bacteria returns.

In summary, all Australian medical professionals that manage pregnant women must be aware that gentamicin is safe to use in pregnancy. When used correctly 
in a timely manner undeterred by its drug categorisation, it can have a significant positive impact on sepsis outcomes in the pregnant woman and her fetus.

\section{References:}

1. Ward K, Theiler RN. Once-daily Dosing of Gentamicin in Obstetrics and Gynecology. Clin Obstet Gynecology. 2008 Sep;51(3):498-506.

2. Kirkwood A, Harris C, Timar N, Koren G. Is gentamicin ototoxic to the fetus? J Obstet Gynaecol Can. 2007 Feb;29(2):140-5.

3. Kumar A, Zarychanski R, Light B, Parrillo J, Maki D, Simon D, et al. Early combination antibiotic therapy yields improved survival compared with monotherapy in septic shock: A propensity-matched analysis*. Crit Care Med. 2010 Sep;38(9):1773-85.

4. Turnidge JD, Gottlieb T, Mitchell DH, Coombs GW, Daly DA, Bell JM, et al. Community-onset Gram-negative Surveillance Program annual report, 2012. Commun Dis Intell Q Rep. 2014 Mar;38(1):E54-8.

5. Czeizel AE, Rockenbauer M, Olsen J, Sørensen HT. A teratological study of aminoglycoside antibiotic treatment during pregnancy. Scand J Infect Dis. 2000;32(3):309-13.

6. Locksmith GJ, Chin A, Vu T, Shattuck KE, Hankins GDV. High Compared With Standard Gentamicin Dosing for Chorioamnionitis: A Comparison of Maternal and Fetal Serum Drug Levels. Obstet Gynecol. 2005 Mar;105(3):473-9.

7. Federal Registration [Internet]. Dec 4, 2014 pp. 72064-103. Available from: https://www.gpo.gov/fdsys/pkg/FR-2014-12-04/pdf/2014-28241.pdf 
Use of Gentamicin in Pregnancy: The 'alphabets' are not elementary

Author Details:

R. Chean ${ }^{1,2}$, S.M. Garland $3,4,5,6$, L. Leung 7

${ }^{1}$ Infectious Diseases physician, Department of Medicine, Latrobe Regional Hospital

2 Department of Microbiology, Dorevitch Pathology

${ }^{3}$ Department of Microbiology and Infectious Diseases, Royal Women's Hospital

${ }^{4}$ Department of Microbiology, Royal Children's Hospital

${ }^{5}$ Murdoch Childrens Research Institute

${ }^{6}$ Department of Obstetrics and Gynaecology, University of Melbourne, Parkville

${ }^{7}$ Department of Pharmacy, Royal Women's Hospital

\section{Author Correspondence:}

Dr Roy Chean

Email Address: royale168@hotmail.com

Postal Address: Dorevitch Pathology, 18 Banksia Street, Heidelberg VIC 3084

Telephone Number: 0412000763

Acknowledgements: Nil Applicable

Word Count Abstract: 158

Word Cont of Main Text: 500

This article is protected by copyright. All rights reserved. 


\section{Abstract:}

Gentamicin has a very important role in the treatment of sepsis including in obstetrics patients. We postulate that the use of gentamicin is overcautious in pregnant women in Australia due to its drug categorisation in pregnancy of 'D'. This designated risk categorisation is due to irreversible congenital deafness in children on streptomycin and small animal studies suggesting nephrotoxicity from gentamicin, but serious adverse effects attributed to gentamicin administered during pregnancy have rarely been documented.

Recent changes by the US FDA to abolish the pregnancy categories labelling of A, $\mathrm{B}, \mathrm{C}, \mathrm{D}$, and $\mathrm{X}$ of human prescription drugs and biological products will allow medical practitioners and patients to make better-informed decisions regarding the use of medicines during pregnancy. This change will also help clarify the role of gentamicin and demystify its potential safety concerns in antenatal sepsis. We advocate that gentamicin is an effective and safe antibiotic when used correctly and in a timely manner in septic obstetrics patients.

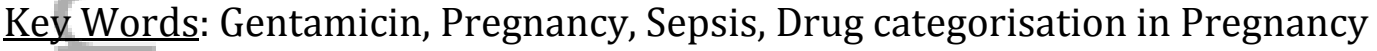

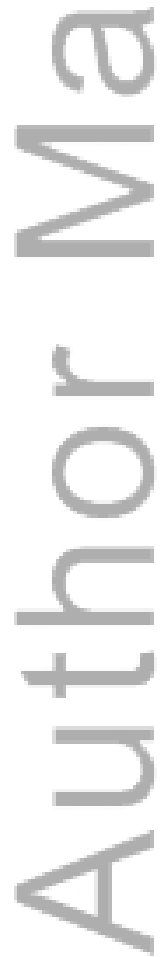




\section{University Library}

\section{- M M I E E R VA A gateway to Melbourne's research publications}

Minerva Access is the Institutional Repository of The University of Melbourne

Author/s:

Chean, R;Garland, SM;Leung, L

Title:

Gentamicin in pregnancy: seeing past the drug categorisation in pregnancy

Date:

2017-01-01

Citation:

Chean, R., Garland, S. M. \& Leung, L. (2017). Gentamicin in pregnancy: seeing past the drug categorisation in pregnancy. INTERNAL MEDICINE JOURNAL, 47 (1), pp.124-125. https://doi.org/10.1111/imj.13315.

Persistent Link:

http://hdl.handle.net/11343/292278 